\title{
THE QUAY CRANE DEPLOYMENT PROBLEM AT A MARITIME CONTAINER TERMINAL
}

\author{
Pasquale Legato \\ Dipartimento di Elettronica, Informatica e Sistemistica \\ University of Calabria \\ Via P. Bucci, Cubo 41C, 87036, Rende (CS), Italy \\ E-mail: legato@deis.unical.it
}

\author{
Daniel Gullì, Roberto Trunfio \\ Centro di Supercalcolo per l'Ingegneria Computazionale \\ CESIC - NEC Italia S.r.l. \\ Via P. Bucci, Cubo 22B, 87036, Rende (CS), Italy \\ E-mail: \{daniel.gulli, roberto.trunfio\}@eu.nec.com
}

\section{KEYWORDS}

Quay crane deployment problem, scheduling problem, maritime container terminals, discrete-event simulation, optimization.

\begin{abstract}
Container unloading/loading at marine container terminals (MCTs) is a key logistic process, to which some research efforts have been addressed by using mathematical programming models formulated in a deterministic-static environment. Vice versa, DES models in a stochastic-dynamic environment are well capable of representing the entire process. Hence, simulation results to be an effective planning and control tool for decision making at all decisional levels. Here we remark that optimal decisions in MCTs may be practically pursued by modelling the whole MCT and focusing attention on the core logistic processes, while representing in a simplified manner the remainder. We focus on the operational management of the cranes deployed along the quay, during the container unloading/loading process at a given number of vessels according to a previously planned berthschedule. We suggest a two-phase approach to the quay crane deployment problem: in the first phase an IP model is used to decide when and how many cranes must be assigned to each vessel; afterwards, we propose a heuristics to determine which specific crane should be assigned to a vessel. We indicate how this approach can be successfully integrated in a DES model, already available, to support dynamic assignment of cranes to berthed vessels.
\end{abstract}

\section{INTRODUCTION}

Freight transportation plays a key role in modern economies as it allows goods exchange between far-off countries. The most notable and steady technology for transporting freight, especially on long maritime routes, is containerization. A considerable growth in worldwide containerised freight transportation has been registered in recent years - approximately 90 percent of the world's cargo traffic moves by container (UNCTAD 2007).

Competitiveness within the growth can be achieved by enforcing the introduction of decision support systems in the organization and management of core logistic processes involved in transportation. An efficient and effective management of logistic activities in a container terminal can decrease the operating costs and service times and increase the quality of services.

A maritime container terminal is a complex facility organised around a set of logistic processes. The logistic activities at a container terminal often belong to more complex logistic processes. This fact is critical for a good management of the system and the choice of the system modelling approach. A firm classification of the decision problems in a maritime container terminal concerns to the following logistic processes (Vis and De Koster 2003; Steenken et al. 2004): i) arrival of the ship, ii) unloading and loading of the ship, iii) transport of containers from ship to stack and vice versa, iv) stacking of containers, and v) inter-terminal transport and other modes of transportation.

Several interesting papers focusing on the previous logistic processes have been proposed (Legato and Mazza 2001; Park and Kim 2003; Legato and Monaco 2004; Cordeau et al. 2005; Cordeau et al. 2007; Canonaco et al. 2008). Among these works, only the ones that are based on a simulation modelling approach, to capture the dynamic and non deterministic framework, are able to evaluate large instances in a reasonable time, to conduct scenario analysis and overall performance evaluation. Besides, simulation offers the opportunity of highlighting congestion phenomena occurring at those (shared) resources resulting as bottleneck within a logistic process (e.g. the quay cranes).

In this paper we propose a two-phase approach to the quay crane deployment problem (QCDP). The QCDP is a complex scheduling problem that arises when multiple vessels berths to a quay and a limited set of quay cranes must be assigned to the berthed vessels in order to respect vessels committed due-time of departure. The first phase consists of an integer programming model that produces the optimal number of cranes that must be assigned to each berthed vessels on the basis of a one-hour time-slot. In the second phase, this output data are used to assign the cranes to the vessels according to an ad hoc heuristics.

We are currently integrating the proposed approach in a discrete-event simulator to support runtime crane assignment using a deterministic berth schedule. The approach has been validated using the Park and Kim 
(op cit) schema. The comparison study showed that our approach minimizes the number of utilized cranes and maximizes the cranes utilization. It also guarantees the vessel overall completion time and the respect of noncrossing constraints for rail-mounted quay cranes.

The remainder of this paper is organized as follows. For sake of completeness, in the next section we briefly describe the logistic processes in which our approach is arranged in. Afterwards we focus our attention to the QCDP and then we define the mathematical model and the heuristics applied within the two-phase approach. At last, we show some computational results to illustrate the potentiality of our approach.

\section{PROBLEM DESCRIPTION: LOGISTIC PROCESSES AND MODEL OBJECTS IN A MARINE CONTAINER TERMINAL}

Modelling maritime container terminal is a complex activity. Simulation is the most powerful tool for the study of this complex and large system, but specific algorithm must be used to model complex processes and to solve assignment and scheduling problems: on that way, mathematical models and heuristics helps in the best modelling of the system. In the following, we first identify the set of model objects involved into a container terminal and then we briefly describe the logistic processes that are involved into the QCDP.

A marine container terminal is a large facility composed by: an harbor entrance wide and water-depth enough followed by an adequate roadstead where incoming vessels may stop and wait; a small number of pilot boats; one or more quay of an adequate length (generally more than $1,000 \mathrm{~m}$ ) with along a channel of multiple water depth ranging; for each quay, a limited set of rail-mounted gantry cranes (RMGs) and rarely less rubber-tyre gantry cranes; a fleet of straddle carriers (SCs) or AGV shuttle vehicles (AGVs) used to transfer containers between the quay cranes and the yard (SVs are able to handle containers if there are no yard cranes); a set of RMGs for the yard operations; and a large yard surface suitably organized in sectors by a regular layout (yard from large container terminals are able to store nearly thousands twenty equivalent units - better known as TEUs, an ISO standardized containers unit measure).

The logistic processes at a container terminal are described in the following.

When calling at the port, a vessel makes a sort of "advanced reservation" based on its ETA - Expected Time of Arrival. Ship entrance into the port depends on the following requirements: i) formal conditions (e.g. contractual agreements between the ship's shipping line and the port of call for the use of port facilities), that means a priority policy for the port entrance queue; ii) operational settings (i.e. pilot boat availability, berth spaces assignment). If requirements are met, the ship is maneuvered into its berth slot by one or two pilot boats; otherwise it must wait in the roadstead. This is the so called arrival of the ship process. Vessels are of two types: mother vessels and feeders. The first one is a large container ship (up to 3,000 TEUs and no more than 14,000 TEUs) that covers transoceanic lines (hub-to-hub connections). Feeders are smaller ships that cover short and middle routes. They are widely used to connect the spokes to the transshipment hub (and vice versa).

Once a vessel is berthed, container discharge/loading can be initiated only if mechanical (and human) resources are allocated; if not, the ship waits in its berth position until resource assignment. Discharge/loading operations are performed by RMGs placed along the berth: one or multiple cranes move containers between the ship and the quay area. The maximum number of quay cranes that may be assigned to each vessel is restricted by $i$ ) the total number of cranes in the quay and ii) the maximum number of allowed cranes for each vessel, due to physical (i.e. the length of the vessel) and logical constraints (i.e. interference between cranes operations). Considering the span of the cranes (approximately $30 \mathrm{~m}$ ) and the horizontal space necessary to stack and to transfer away the incoming/outgoing containers of a vessel, the maximum number of cranes allowed for the longest vessel is usually 5 (this number is proportionally decreased for shortest vessel). When multiple cranes are assigned to the same ship, crane interference has to be avoided and a complex scheduling problem arises to manage the relationships (precedence and mutual exclusion) existing among the holds of the same ship. Considering that the service rate of the RMGs is 30 TEUs/hour, the performance of the discharge/loading process highly depends on the availability of this type of cranes and their turnover speed. Therefore, the best deployment of these resources affects the overall completion time of each vessel. Finally, a fleet of vehicles, SCs (or AGVs), take in charge containers and move forth and back between the berth area and the yard area.

\section{The Quay Crane Deployment Problem}

In the unloading and loading of the ship process, a key decisional problem is the Quay Crane Deployment Problem (QCDP). This problem has been successfully discussed by Park and Kim (2003) together the berth scheduling problem. The QCDP is described in the following.

Planners of the terminal operating company weekly construct a "berth schedule", which shows the berthing position and time windows for the incoming vessels (solution to the so called berth scheduling problem). A time window shows the expected time of berthing and un-berthing for each vessel; time windows are constructed using the ETA (Expected Time of Arrival) and PTD (Promised Time of Departure) of each vessel (a penalty cost is incurred if the departure of a vessel is later than the previously committed PTD). In Figure 1 is shown an example of berth schedule, where the 
berth-time and space have been partitioned into $22 \times 24$ grid squares (24 time-slots of one hour).

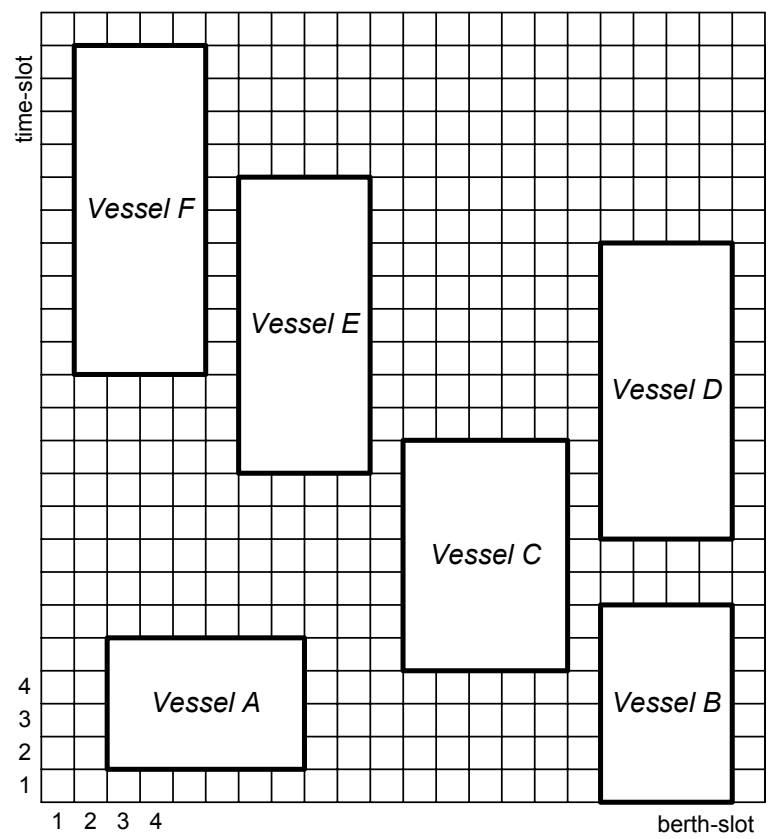

Figure 1: An Example of Berth Schedule Presented by Park and Kim

In the QCDP, the berth schedule is used to assign the RMG cranes to the incoming vessels on a weekly basis. The double goal is i) minimize the number of used quay cranes and to ii) maximize their utilization, under the constraint of completing the loading/unloading operations, for each vessel, within their expected time of un-berthing,

The QCDP is solved under the following assumptions:

1. Each vessel has a time window; the start time of the time window is the expected time of berthing of the vessel, while its end is the vessel expected time of un-berthing.

2. Each vessel has a total number of TEUs to be handled in its time window: this number is put in correspondence with the moves required by the vessel

3. Each vessel has a maximum and a minimum number of cranes that can and must be assigned when the operations starts. The maximum number of cranes that can be simultaneously assigned to a vessel is limited by vessel length. Vice versa, the minimum number of cranes to be assigned is due (usually for mother vessels) to contract terms between terminal operating company and the vessel shipping company. By default, when the operations start on a vessel, until the operations are completed all the time-slots related to the vessel have almost one assigned crane.
4. Quay cranes are of the RMG type, so there are nocrossing constraints that must be guaranteed. Furthermore, cranes are never un-available.

The solution approach we propose here to the QCDP is decomposed in two phases, as shown in Figure 2.

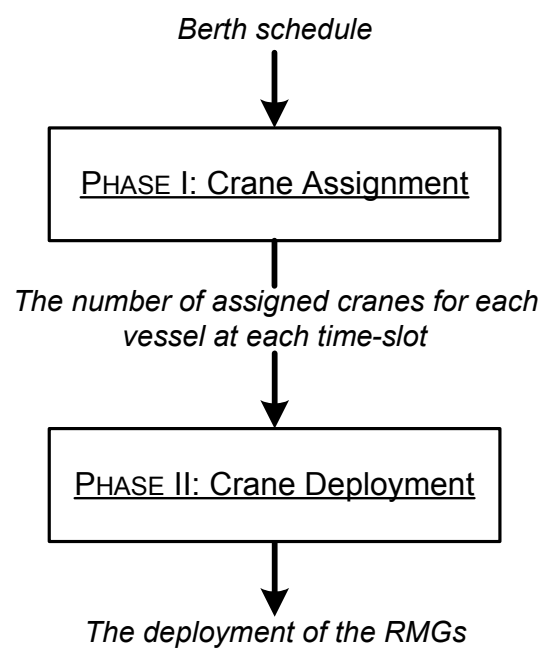

Figure 2: The Schema of the Two-Phase Approach to the QCDP

In the first phase, we solve a mathematical model using CPLEX solver (ILOG 1999) to identify the optimal number of cranes that must be assigned to each vessel for each time-slot. Thus, the model is able to identify exactly when the discharging/loading operations starts and ends within the vessel timewindow.

In the second phase, an ad hoc built heuristics is used to assign the cranes to the vessels in order to respect non-crossing constraints and to minimize the number of crane shifts from a vessel to another.

\section{An IP Model for The Crane Assignment Phase}

The following notations will be used for the formulation of the IP model used in the crane assignment phase (i.e, the Quay Crane Assignment Problem - QCAP):

T : The set of time-slots, with $|\mathrm{T}|=\mathrm{N}$

$\Omega \quad$ : The set of vessels

C : The set of quay cranes, with $|\mathrm{C}|=\mathrm{M}$

$s_{c}:$ : The service rate for the $c$-th crane, expressed in TEUs per time-slot

$m_{i} \quad$ : The number of moves for the $i$-th vessel

$e t b_{i} \quad$ : The berthing time for the $i$-th vessel, i.e. the time-slot starting from which the vessel $i$-th is ready, at the berth, for the first lift, where:

$$
1 \leq e t b_{i} \leq N-e t u_{i}+1
$$

$e t u_{i} \quad$ : The un-berthing time of the $i$ - $t h$ vessel; thus it is the latest time-slot until 
which the vessel $i$-th is available for the operations, where:

$$
e t b_{i} \leq e t u_{i} \leq N
$$

$\min _{i} \quad$ : The minimum number of cranes that must be assigned to the $i$-th vessel, when the operation starts.

$\max :$ The maximum number of cranes that $i \quad$ can be assigned to the $i$-th. vessel

We introduce the following decisional variables:

$V \quad:$ The maximum number of cranes used to performs vessels operations.

$\theta_{i t}^{c}: 1$, if crane $c$ works on the vessel $i$ at the time-slot $t, 0$ otherwise.

$\phi_{i t}: \quad 1$, if vessel $i$ is processed at time-slot $t$, 0 otherwise.

$\gamma_{i t}: 1$, if operations for the vessel $i$ were started at time-slot $t, 0$ otherwise.

$\eta_{i t}: 1$, if operations for vessel $i$ have not been completed at time-slot $t, 0$ otherwise.

The QCAP can be formulated as follows:

$$
\begin{aligned}
& \min N \cdot V+\sum_{i \in \Omega} \sum_{t=e t b_{i}}^{e t u_{i}} \gamma_{i t}+\sum_{i \in \Omega} \sum_{t=e t b_{i}}^{e t u_{i}} \eta_{i t} \\
& \text { s.to } \\
& \gamma_{i t} \leq \gamma_{i(t+1)} \quad \forall i \in \Omega, t=e t b_{i}, \ldots, e t u_{i} \\
& \eta_{i t} \geq \eta_{i(t+1)} \quad \forall i \in \Omega, t=e t b_{i}, \ldots, e t u_{i} \\
& \gamma_{i t}+\eta_{i t}=\phi_{i t}+1 \quad \forall i \in \Omega, t=e t b_{i}, \ldots, e t u_{i} \\
& \sum_{c \in C} \sum_{t=e t b_{i}}^{e t u_{i}-1} s_{c} \theta_{i t}^{c}=m_{i} \quad \forall i \in \Omega \\
& \sum_{c \in C} \sum_{i=1}^{e t b_{i}-1} \theta_{i t}^{c}=0 \quad \forall i \in \Omega \\
& \sum_{c \in C} \sum_{t=e t u_{i}+1}^{n} \theta_{i t}^{c}=0 \quad \forall i \in \Omega \\
& \sum_{i \in \Omega} \theta_{i t}^{c} \leq 1 \quad \forall c \in C, t \in T \\
& \sum_{c \in C} \theta_{i t}^{c} \leq \max _{i} \phi_{i t} \quad \forall i \in \Omega, t=e t b_{i}, \ldots, e t u_{i} \\
& \sum_{c \in C} \theta_{i t}^{c} \geq \min _{i} \phi_{i t} \quad \forall i \in \Omega, t=e t b_{i}, \ldots, e t u_{i} \\
& \sum_{c \in C} \sum_{i \in \Omega} \theta_{i t}^{c} \leq V \quad \forall t \in T \\
& V \geq 0 \text {, int } \\
& \theta_{i t}^{c} \in\{0,1\} \\
& \forall i \in \Omega, t \in T, c \in C \\
& \phi_{i t}, \gamma_{i t}, \eta_{i t} \in\{0,1\} \quad \forall i \in \Omega, t \in T
\end{aligned}
$$

\section{A Heuristic Approach for the Crane Deployment Phase}

As stated before, the solution of the mathematical model provides the number of cranes that must be assigned in order to complete the operations in time. This data are used in the following to assign, for each time-slot, the RMGs.

The step-by-step description of our heuristics is given in the following.

\section{Step 0. Initialization phase}

Select V contiguous quay cranes from the set C, thus let $Q$ be the set of selected quay cranes ordered by non decreasing position along the quay. Let $S$ be the number of berthslots and $M$ be the number of the first half of berth-slots $(M=\lceil L / 2\rceil)$; besides, let from $_{i}$ the berth-slot starting from which the vessel $i$ is berthed and $c_{i t}$ be the number of quay cranes assigned to the vessel $i$ at the time-slot $t$.

Step 1. Crane assignment phase FOR EACH $t \in T$

Let $B$ be the set of the berthed vessels; Compute $c_{t}$ as the sum of all the $c_{i t}$;

Let $k=|Q|-c_{t}$ be the number of cranes that will be idle during the time-slot $t$; IF $" B \neq \varnothing "$ THEN

Order $B$ by non-decreasing order of berth position;

Extract the vessel $j$ from the top of $B$;

$$
\begin{gathered}
I F \text { " } \text { from }_{i}<M " \text { THEN } \\
\text { WHILE "B } \neq \varnothing " D O
\end{gathered}
$$

i. Extract the vessel $i$ from the top of $B$;

ii. IF operations for the vessel $i$ were started at time-slot $t-1$ $\left(\phi_{i(t-1)}=1\right)$ THEN

IF $k>0$ THEN

Extract no more than $k$ cranes from the top of $Q$ if none of this crane was already assigned to the vessel $i$; Update k;

END IF

END IF

iii. Extract $c_{i t}$ cranes from the top of $Q$ and assign them to the vessel $\mathrm{i}$; 


\author{
END WHILE \\ ELSE \\ WHILE " $B \neq \emptyset^{\prime \prime} D O$ \\ i. Extract the vessel $i$ from the \\ bottom of $B$; \\ ii. IF operations for the vessel $\mathrm{i}$ \\ were started at time-slot $t-1$ \\ $\left(\phi_{i(t-1)}=1\right)$ THEN

\section{IF $k>0$ THEN} \\ Extract no more than \\ $k$ cranes from the \\ bottom of $Q$ if none \\ of this crane was \\ already assigned to \\ the vessel I \\ Update k; \\ END IF \\ END IF \\ iii. Extract $c_{i t}$ cranes from the \\ bottom of $Q$ and assign them \\ to the vessel $\mathrm{i}$; \\ END IF \\ END WHILE \\ END FOR
}

\section{A Numerical Experiment}

At this point, we compare the results obtained using our approach against the results showed by Park and Kim (2003). In their work, a method for constructing a berth schedule and then to deploy cranes was also developed. Therefore, we compare their results with ours to demonstrate the validity of our method.

In Figure 3 we repeat the optimal assignment reported into the paper by Park and Kim based on the berth schedule previously shown in Figure 1. For each vessel, ringed numbers depict the quay cranes assigned to each vessel. As it is possible to see, the QCDP solved by Park and Kim use 9 cranes to complete all the operations in time.

In this case study, the minimum number of cranes that must be assigned to the vessels during the operations is one, while the maximum number of cranes that is possible to assign to a specific vessel is equal to the number of occupied berth-slots.

The first step of our approach produces the assignment depicted in Figure 4. In this berth schedule, for the $i$-th vessel and the $t$-th time-slot the optimal $c_{i t}$ is shown.

As it is easy to recognise, our mathematical model fills a berth schedule minimizing the overall number of cranes that must be used to process all the vessels. In fact, the first phase of our approach produced an assignment of 7 quay cranes against the 9 quay cranes found by Park and Kim. Moreover, in our solution, when the operations start there is no operations discontinuity for each vessel: this is a primary contractual agreement requested to the terminal operating company by shipping companies as well as the minimum number of assigned cranes and the respect of the committed operations completion time.

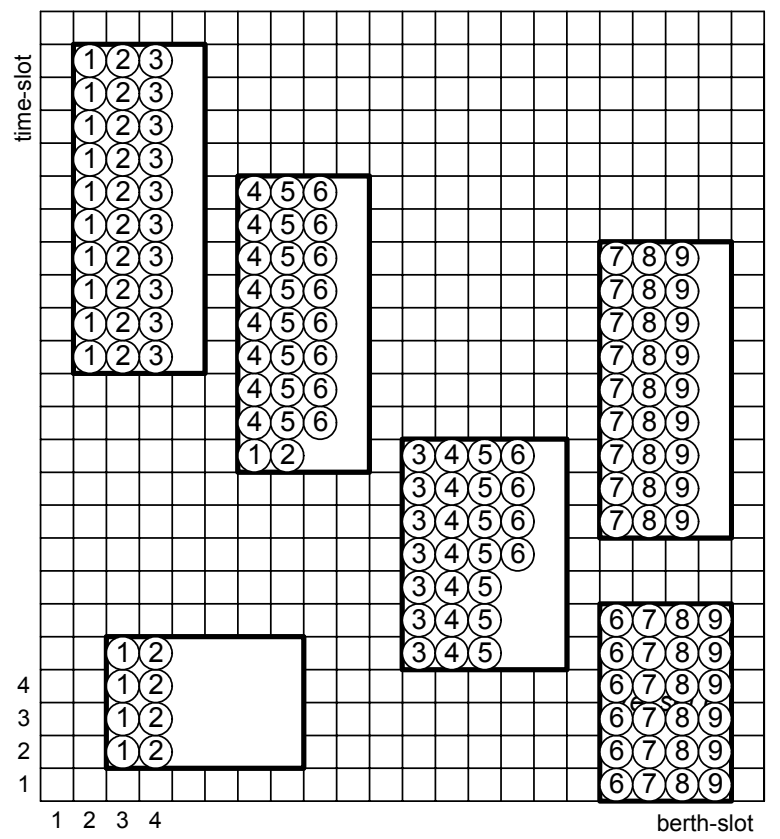

Figure 3: The Optimal Deployment Found by the Park and Kim Methodology

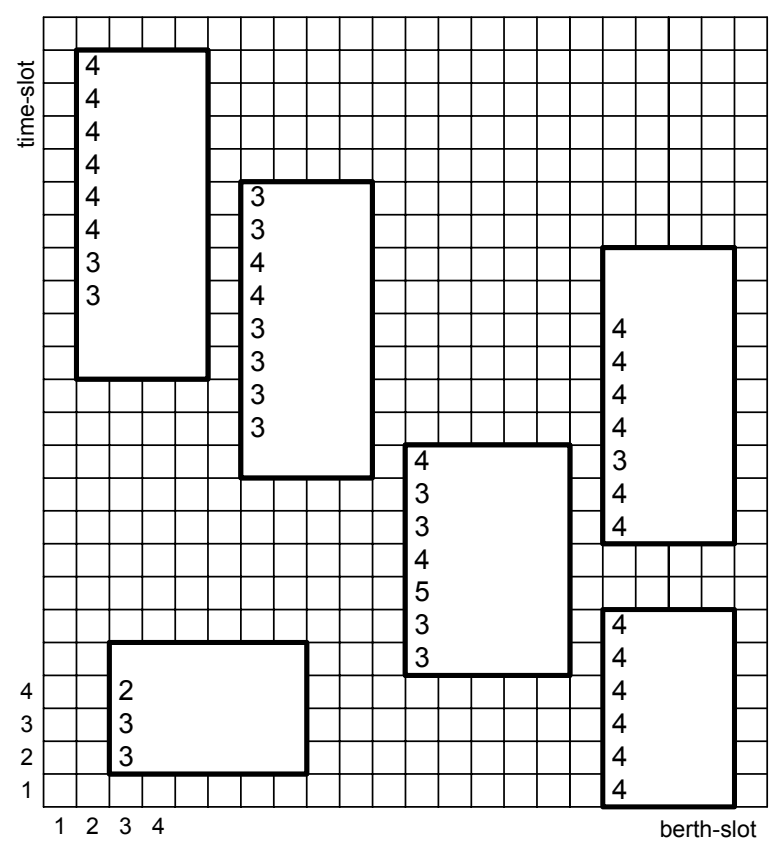

Figure 4: The First Phase Output of our Methodology

As results of the second phase of our algorithm, we propose the quay cranes deployment shown in figure 5 .

The improvement obtained with the approach proposed in this paper is not only due to the minimization of the activated cranes. In fact, $i$ ) in two cases we obtained a reduction of the vessel overall 
completion time and ii) we improved the average crane utilization (0.69 vs. 0.87).

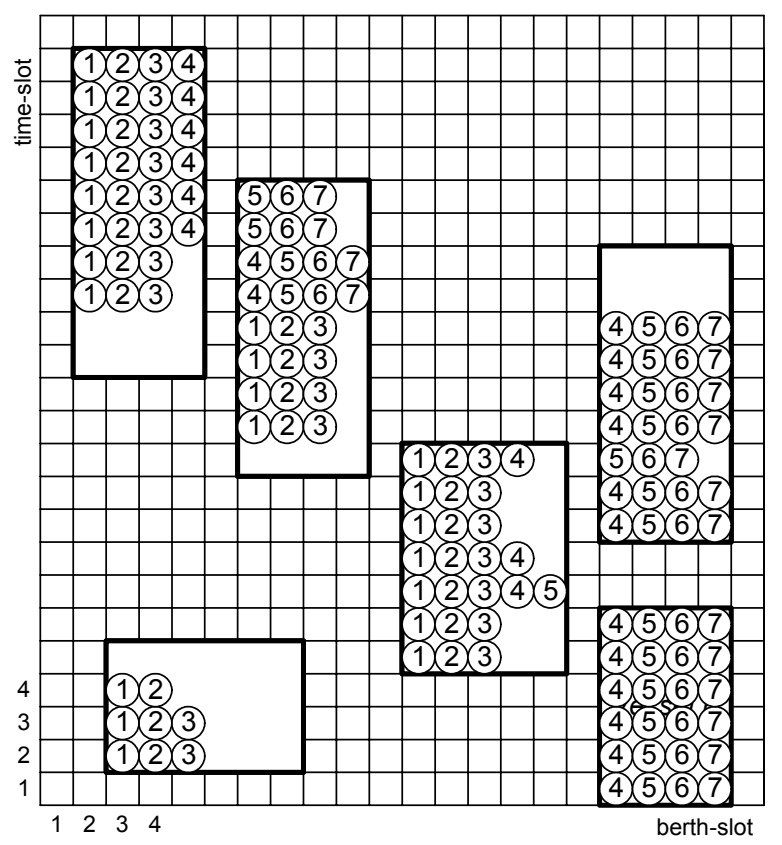

Figure 5: The Final Output by our Methodology

\section{THE NEED FOR SIMULATION INTEGRATION}

As stated in the introduction, our belief is that deterministic approaches must be integrated into simulation platforms to conduct scenario analysis through statistical estimates of some system performance measures (Nakayama 2002).

Currently, we are integrating this methodology into a discrete-event simulator for the berth planning and discharge/loading processes at a real maritime container terminal. As a matter of fact, the starting point is the simulator validated and tuned for one of the operating company of the Gioia Tauro terminal, located in the southern Italy (Canonaco et al. 2008). The terminal is the most important transhipment terminal in the Mediterranean Sea and our integrated model will allow the planners of the terminal to identify the best cranes deployment day-per-day. The role of the simulator is central due its capability of verifying the practical possibility of guaranteeing the respect of the planned time windows (berthingunberting), in a more realistic modelling environment where operation delays and unpredictable events may occur,

As guideline for the integrated simulator, observe that the programmed time-instants of berthing and unberthing, for each vessel, are considered as fixed events within the events list of the simulator. Vice versa, all the operations involved in the dischargingcharging process of whatever vessel will be represented with their natural randomness. Particular attention will be devoted to capture the effect of the time required by the process of shifting cranes from one berth slot to another. This time is at the basis of possible delays upon the programmed time instants of unberthing. Whenever crane shift operations should reveal to be cumbersome and/or time consuming, the terminal manager is asked to evaluate the possibility of increasing the number of available cranes suggested by our two step methodology.

Finally, we are also addressing the problem of integrating a second integer programming model aimed to schedule the work of those cranes assigned to a specific vessel with respect to the stows to be handled. Stow processing requires some precedence constraints and non simultaneous processing of adjacent stows by two different cranes. Thus an adequate crane scheduling policy should be embedded into the simulator, besides the crane deployment one.

\section{CONCLUSION}

We have proposed a methodology for the Quay Crane Deployment Problem. The methodology consists of a mathematical programming model and a heuristics. The mathematical model is used to identify the optimal number of quay cranes that must be assigned to the vessel time by time. The heuristics is used to identify which cranes must be assigned to the vessel time by time. We have compared our method with the optimal results obtained by another promising work. The comparison proved that our approach is able to outperform the previous near-optimal solution. Thus, we propose to embed our method into a simulation model to perform scenario analysis at the operational level in order to verify whether or not the time requirements of each vessel can be guaranteed by a suitably fixed number of quay cranes. Finally, we are developing alternative simulation based approaches to supply at the restriction implied by integer programming. Simulation-based Optimization looks as the most promising practical technique to support decisions for the Quay Crane Deployment Problem.

\section{REFERENCES}

Canonaco, P.; P. Legato; R.M. Mazza; and R. Musmanno. 2008. "A Queuing Network Model for Management of Berth Crane Operations". Computers \& Operations Research 35, 8, 2432-2446.

Cordeau, J.-F.; M. Gaudioso; G. Laporte; and L. Moccia. 2007. "The Service Allocation Problem at the Gioia Tauro Maritime Terminal", European Journal of Operational Research 176, 1167-1184.

Cordeau, J.F.; G. Laporte; P. Legato; and L. Moccia. 2005. "Models and Tabu Search Heuristics for the Berth Allocation Problem". Transportation Science 39, 4, 526538.

ILOG. 1999. CPLEX 6.5. Mountain View, CA.

Legato, P. and R.M. Mazza. 2001. "Berth Planning and Resources Optimisation at a Container Terminal via Discrete Event Simulation". European Journal of Operational Research 133, 537-547.

Legato, P. and M.F. Monaco. 2004. "Human resources management at a marine container terminal". European Journal of Operational Research 156(3), 769-181. 
Legato, P. and R. Trunfio, 2007. "A Simulation Modelling Paradigm for the Optimal Management of Logistics in Container Terminals". In Proceedings of the 21th European Conference on Modelling and Simulation. (Prague, Czech Republic). 479-488.

Nakayama, M.K. 2002. "Simulation Output Analysis". In Proceedings of the 2002 Winter Simulation Conference. (San Diego, CA, Dec. 8-11). 23-34.

Park, Y.-M. and K.H. Kim. 2003. "A Scheduling Method for Berth and Quay Cranes". OR Spectrum 25, 1-23.

Pidd, M. and R.B. Castro. 1998. "Hierarchical Modular Modelling in Discrete Simulation". In Proceedings of the 1998 Winter Simulation Conference. (Washington, DC, USA). 383-389.

Steenken, D.; S. Voss; and R. Stahlbock. 2004. "Container Terminal Operation and Operations Research - a Classification and Literature Review". OR Spectrum 26, 3-49.

Vis, I.F.A. and R. De Koster. 2003. "Transshipment of Containers at a Container Terminal: an Overview". European Journal of Operational Research. 147(1), 116.

UNCTAD. 2007. United Nations Conference on Trade and Development. Review of Maritime Transport 2007. (United Nations, New York and Geneva).

\section{AUTHOR BIOGRAPHIES}

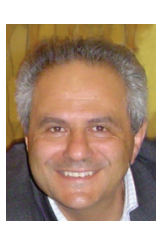

PASQUALE LEGATO is an Assistant Professor of Operations Research at the Faculty of Engineering (University of Calabria), where he teaches courses on simulation for system performance evaluation. He has published on queuing network models for job shop and logistic systems, as well as on integer programming models. He has been involved in several national and international applied research projects and is serving as reviewer for some international journals. His current research activities are on the development and analysis of queuing network models for logistic systems, discrete-event simulation and the integration of simulation output analysis techniques with combinatorial optimization algorithms for real life applications in Transportation and Logistics.

His home-page is http://www.deis.unical.it/legato.

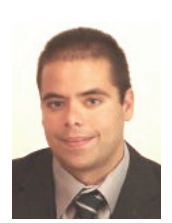

DANIEL GULLÌ received a Laurea degree (cum laude) in Automotive Engineering from the University of Calabria, Rende, Italy, in 2005. He is devoted to research in numerical simulation at NEC Italy Center for High-

Performance Computing and Computational Engineering (CESIC). His current research interests include discrete-event simulation models for logistic systems and parallel simulation.

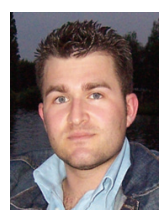

ROBERTO TRUNFIO received a Laurea degree (cum laude) in Management Engineering from the University of Calabria, Rende, Italy, in 2005, and is currently pursuing the Ph.D. degree in Operations Research from the same university. Moreover, he is a logistics engineer at NEC Italy Center for High-Performance Computing and Computational Engineering (CESIC). His current research interests include modelling languages for discrete-event simulation, simulation-based optimisation of logistics systems, and parallel simulation. 